\title{
Surface Vs Volume Based Reconstruction of Bone Tissue Using CAS_Annotate and CAS_Navigate
}

\author{
1,2João Fradinho Oliveira \\ ${ }^{1} \mathrm{C} 3 \mathrm{i} /$ Instituto Politécnico de Portalegre, Portalegre, Portugal; \\ ${ }^{2}$ CIAUD/Faculdade de Arquitectura, Universidade de Lisboa, Lisboa, Portugal; \\ jfoliveira@estgp.pt
}
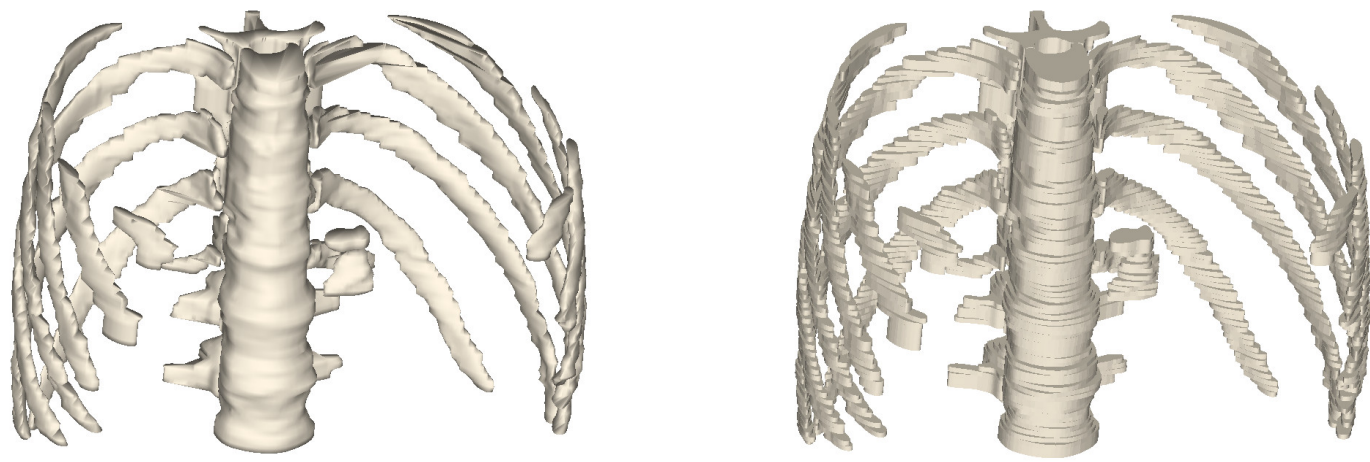

Figure 1-Reconstruction of a pig rib cage left) surface 250412 triangles; right) volume-based 498116 triangles

\begin{abstract}
Intra-operative systems that provide 3D spatial reasoning support, require 3D models whose geometric accuracy enables the surgeon to make relative positioning and orientation decisions of anatomical structures during navigation. This paper compares the advantages and disadvantages of two popular types of 3D reconstruction from CT polygons, surface based and volume based respectively (Figure 1). Both are implemented in the CAS_Annotate tool, and were used for the purpose of assessing bone tissue modeling strategies for navigation. Their impact in rendering performance is assessed in CAS_Navigate which draws on IGSTK's intra-operative visualization capabilities.

Keywords: Bone tissue modeling; Image guided surgery; 3D reconstruction; VTK; IGSTK.

\section{Introduction}

Reconstruction of anatomical structures from planar contours presents some interesting modeling questions such as the contour correspondence and branching problem between polygons on adjacent CT slices [1], in other words, determining when an object starts, ends, joins or splits. The choice of the level of detail modeling which is adequate for a given application is also important to address. Can an object such as a vascular network be represented as a composition of several individual volume blocks?
\end{abstract}


Or does one need an object to be represented as a whole entity, for example to simulate the removal of an entire individual vertebra?. For many applications using vascular structure models such as resection mapping or approximate blood volume calculations, a volume-block reconstruction $[2,3]$ has eliminated all together the task of inferring or correcting connections. Similarly, organs can be a composition of surfaces, together volume-blocks (vascular networks) and composite surfaces (organs) [3] can greatly simplify the modeling task. The question this work addresses, is which of the two reconstruction approaches is preferable for basic bone visualization in an image guided surgery application?.

Before analyzing both approaches, two important factors which are intrinsic to CT and affect both are highlighted. Figure 2, shows the result of manual segmentation of the rib bone structure of a pig, using two different intensity/contrast thresholds. Often a radiologist needs to resort to two settings to infer and delimit a faint contour, making this process time-consuming. Automatic segmentation of anatomical structures in general is a very active area of research, where the comparison of algorithm results on public data sets [4] is essential to further improve and disseminate results.
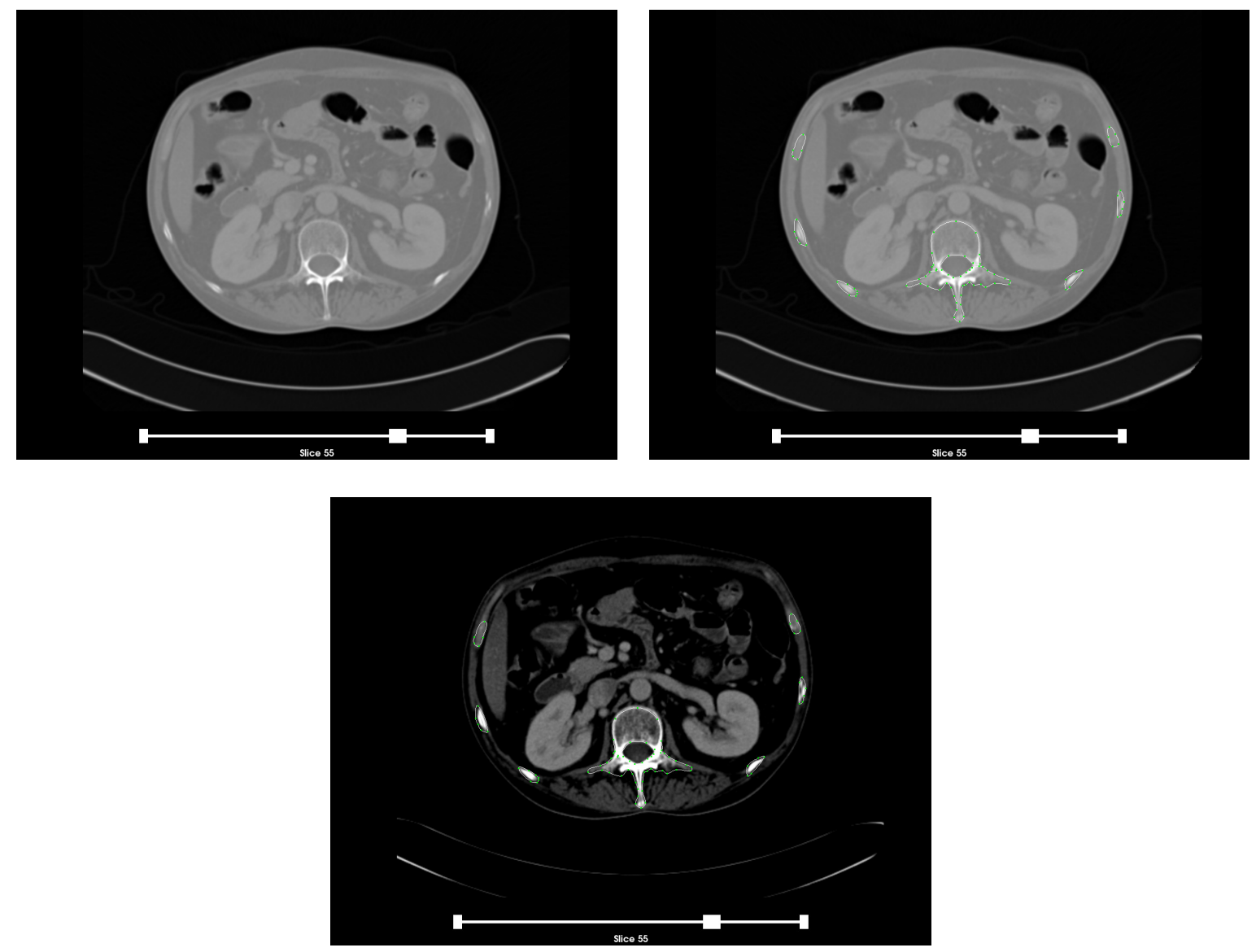

Figure 2 - Segmentation of bone tissue polygon contours with CAS_Annotate. Top left: original slice;

Top right: image threshold T1; Bottom right: image threshold T2

The second factor, is the problem of limited resolution between CT slices. The drastic shape difference between adjacent contours (Figure 3 ) may or may not indicate a new object. 

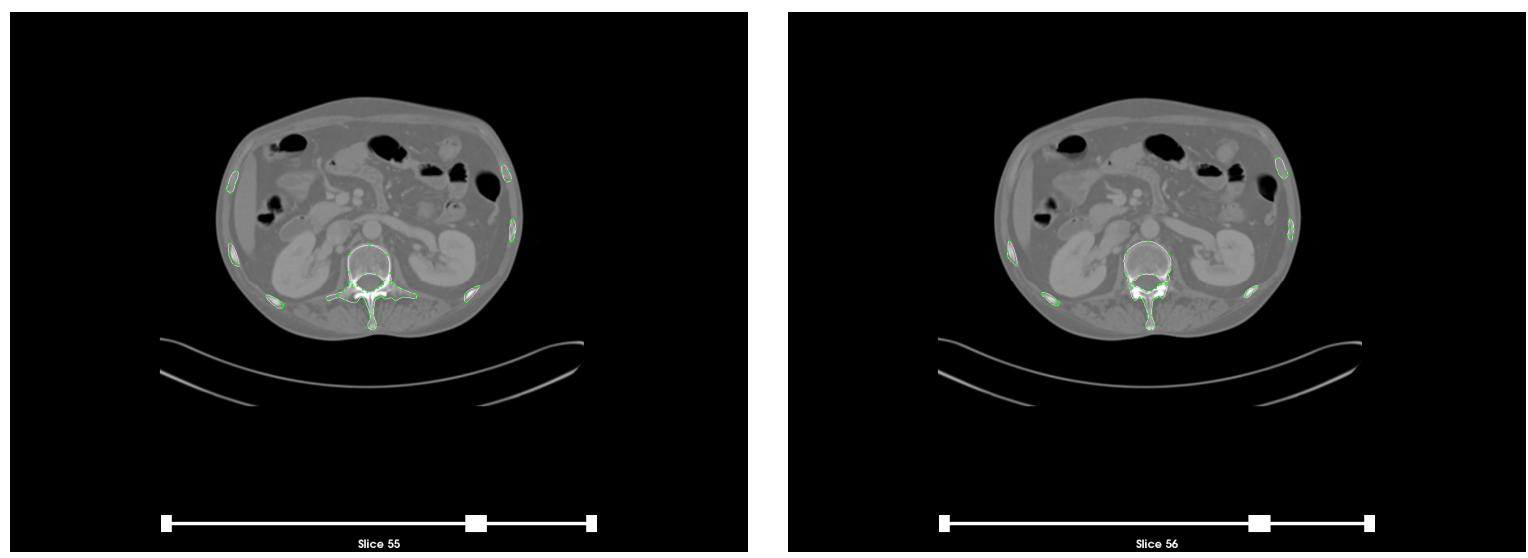

Figure 3 - Drastic shape variation between adjacent slices.

\section{Materials and Methods}

The following diagram (Figure 4) illustrates the main components of the CAS_Annotate and CAS_Navigate tool.

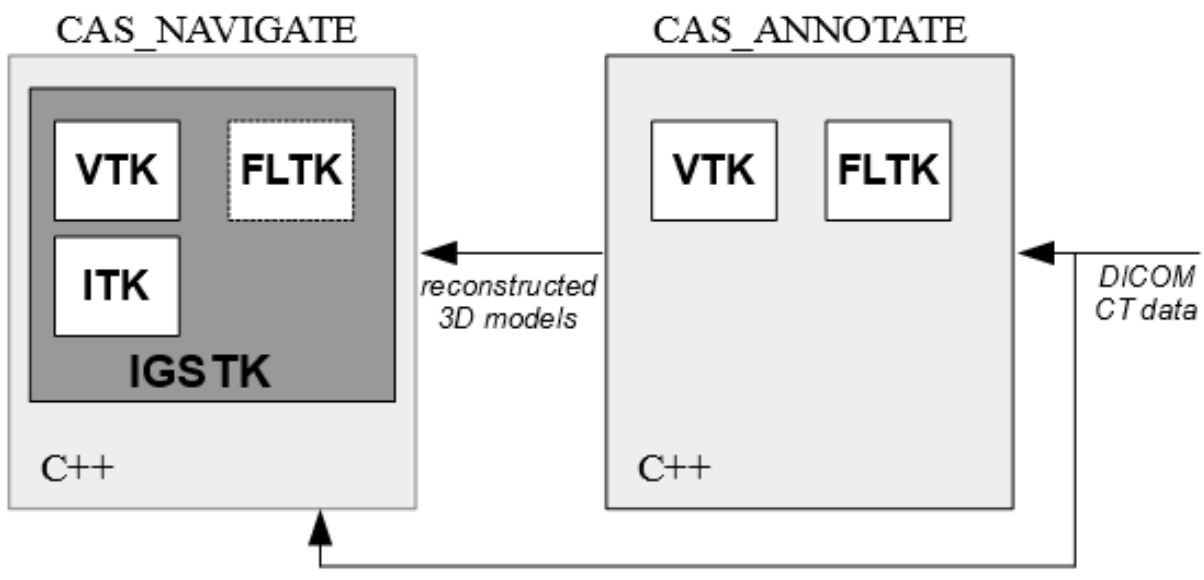

Figure 4 -CAS_Annotate and CAS_Navigate system components

CAS_Annotate uses VTK [5] functionality to: read and display DICOM [6] images (vtkImageViewer2), manually create and edit a polygon contour (vtkContourWidgets). The system uses FLTK [7] to create a user interface that among other functionality allows to select a DICOM image dataset from disk, write and read polygon contours and their correspondences following the file format detailed in [3]. Volumebased reconstruction does not require any polygon correspondences, but surface reconstruction does. Creating a correspondence between two polygons can be done simply by right clicking near a polygon, pressing ' $c$ ', change the slice being viewed, right clicking near the corresponding polygon and pressing ' $v$ '. Once all contours have been created and relevant links established one can choose to create a volume-based reconstruction or a surface reconstruction. With a volume-based reconstruction, every polygon is copied onto the next adjacent slice, with this 1-1 correspondence, vertices from both polygons are simply triangulated and the top and bottom side of the volume block are closed using Eberly's triangulation by ear-clipping algorithm [8]. For the surface reconstruction, the system uses Eberly's triangulation when a polygon contour has no polygon correspondence links either above or 
below. Corresponding polygons in adjacent slices are tilled together using Christiansen and Sederberg algorithm [9] which allows for a different number of vertices to exist in each polygon and also supports an elegant bifurcation solution. Finally, it is also possible to create either a high or low resolution version of a reconstruction, the later extracts only the control points of the spline vtkContourWidgets. For more details please refer to [10]. Once the models have been created, they can be saved in either the PLY format [11] or IGSTK's [12] 3D .Msh object file format.

CAS_Navigate uses IGSTK to read and render efficiently the created 3D models over the DICOM images [13] and to connect surgical trackers.

\section{Results and Discussion}

A Sony Vaio laptop computer with an Intel i7 2.80Ghz processor, 6 GB RAM, and an AMD Radeon 6630M graphics acceleration card was used for the reconstruction and visualization. Whilst the reconstruction of automatically or previously segmented polygons is possible with CAS_Annotate (provided that the polygon vertices follow the contour file layout detailed in [3]), manual segmentation was performed for the polygons in this study. Specifically a 74 slice CT dataset of the rib cage and spine of a pig was chosen, and 715 contour polygons were segmented with a total of 7105 control vertices (some contour polygons are visible in Figure 2 and 3 ).

Low and high resolution models were created for both the surface based reconstruction and the volume based reconstruction. The surface reconstruction, created 19 separate objects using one contour-link file. Incomplete contours, with less than 3 vertices were ignored. Every reconstructed models was created in less than one second. It took approximately 50 minutes to manually establish the contour links, and just under 5 hours to annotate the 715 polygons. Table 1 outlines the model complexity of the four created datasets, the models are shown in Figure 5 with flat rendering on the left column, and wireframe with flat rendering on the right column.

Table 1 - Model complexity

\begin{tabular}{|c|c|c|}
\hline Model & \#Triangles & \#Vertices \\
\hline low_res_block_model & 25472 & 14166 \\
\hline low_res_surface_model & 14090 & 7083 \\
\hline high_res_block_model & 498116 & 250488 \\
\hline high_res_surface_model & 250412 & 125244 \\
\hline
\end{tabular}



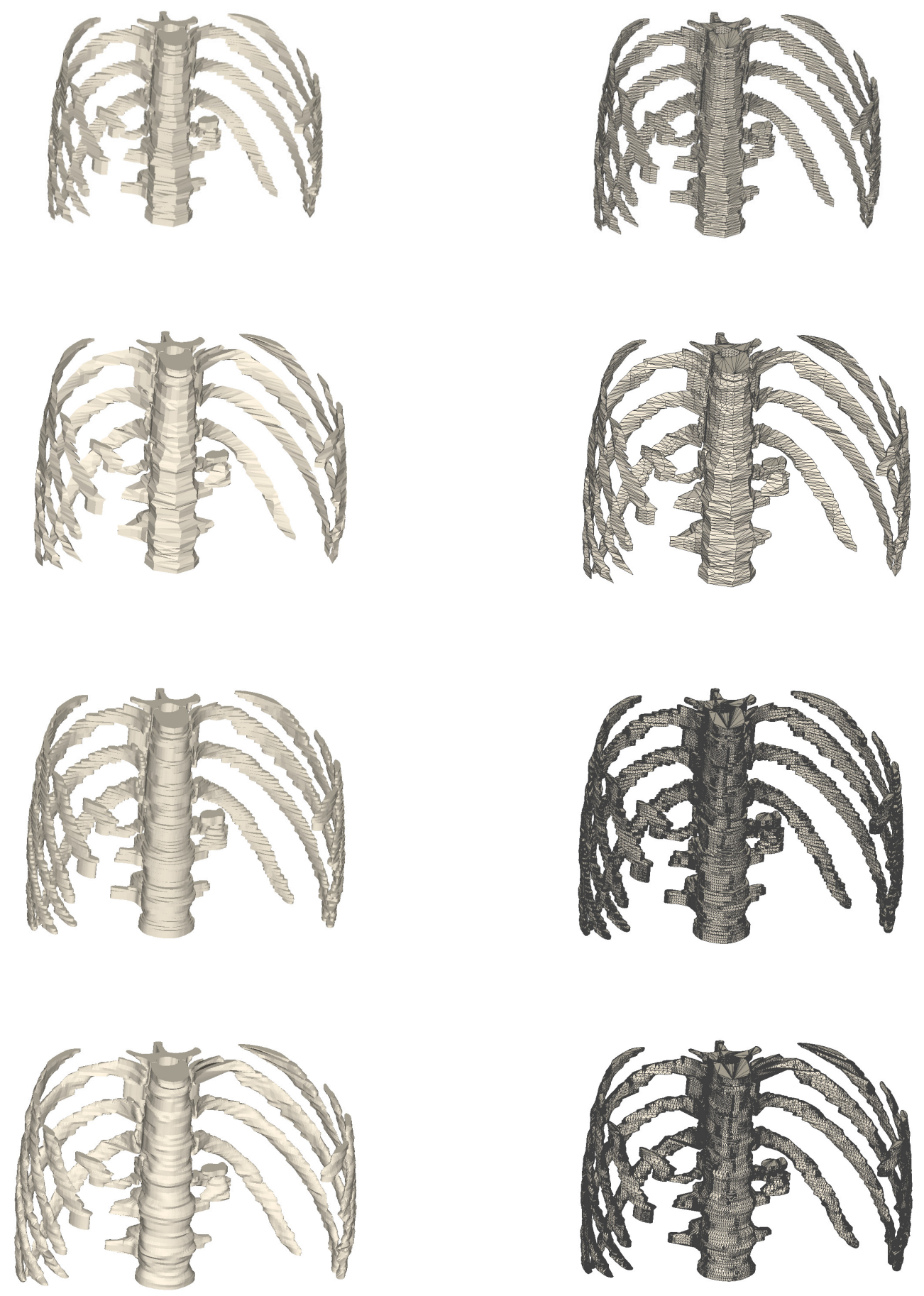

Figure 5 - Model results (left: flat shading, right: flat shading and wireframe rendering) - from top to bottom: low_res_block_model, low_res_surface_model, high_res_block_model, high_res_surface_model.

The first observation of the results, is that the surface based reconstruction generates off-axis geometry which make the created models visually "smooth" in all view angles, whereas the volume-based reconstruction has some view angles where the regular axis aligned structure is more apparent (top row, and third row from top). Another observation is that the higher resolution versions "inflate" the 
models in some areas, more noticeably on the top edge region of the ribs, this occurs as a result of more vertices becoming available to represent the curve. Figure 6 , shows rendering artifacts in the lower left area of the spine on both the low and high resolution surface reconstruction, whilst the volume-based approach on the top right corner has no such artifact. With closer inspection, the artifact results from the surface reconstruction creating long triangles in an effort to stitch two very different polygon contours (as illustrated in Figure 3). One way to alleviate this problem would be to detect during the reconstruction slices that generate long triangles, and generate intermediate intra-slice interpolated contours. It should also be pointed out that the individual vertebra were not modeled as separate objects, rather the spine was created as two continuous structures. Modeling each vertebra individually is a simple matter of not creating links between links before and after the corresponding polygons. However, vertebra do not align with the slices, and it becomes difficult to define when does one begin, and another end.
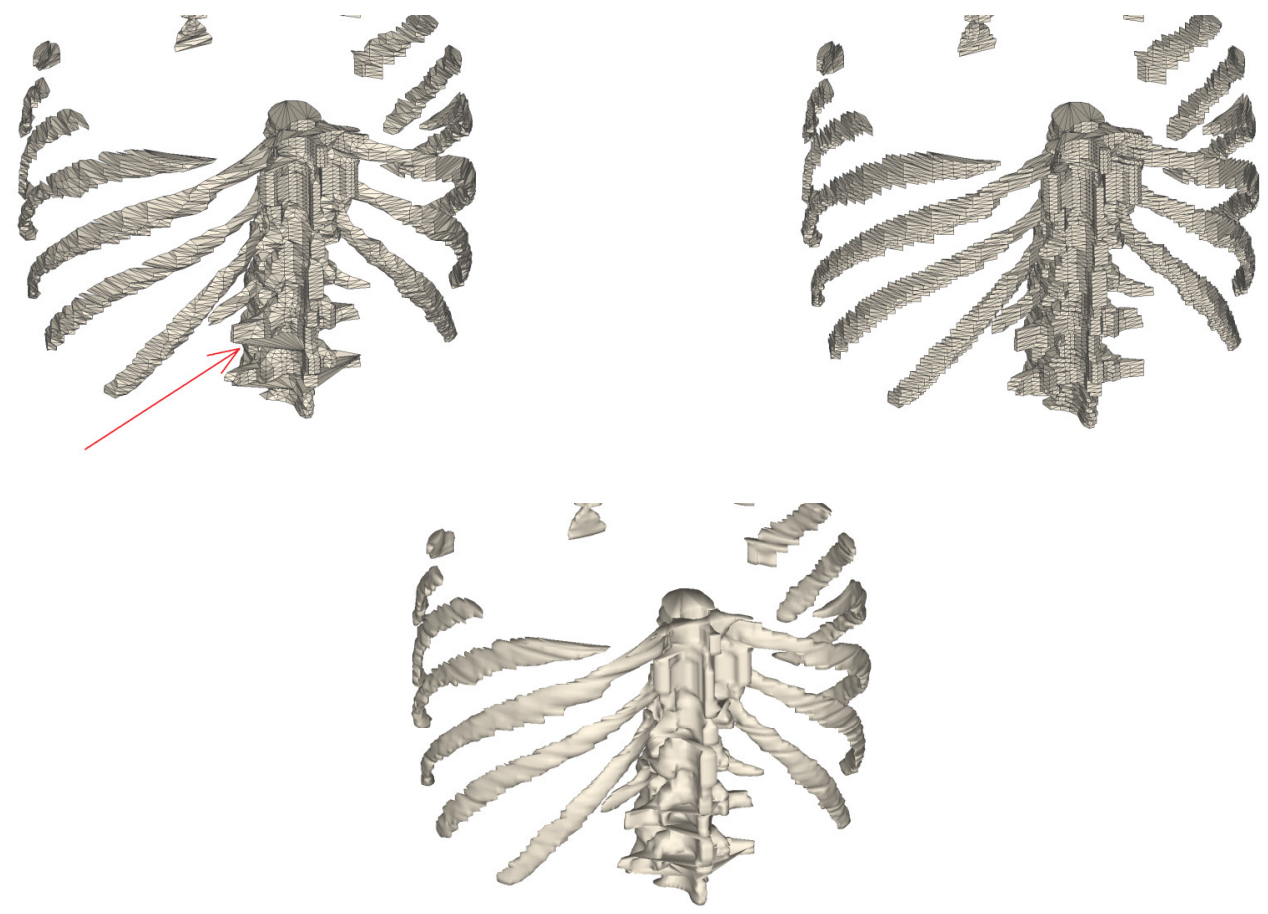

Figure 6 - Drastic shape variation between slices - Surface based reconstruction (left column) Vs Volume based reconstruction (right) - topleft: low_res_surface_model; topright: low_res_block_model; bottom left: high_res_surface_model.

The generated models were loaded in CAS_Navigate, along with models of the liver and vascular structures (Figure 7), and all resolutions (Table 2) rendered at smooth interactive rates. The vascular structures (volume-based reconstruction) and bone models (surface based reconstruction) were set to completely opaque, whilst the organs (surface based reconstruction) had opacity/alpha blending set to 0.4 . 
João Fradinho Oliveira; Surface Vs Volume Based Reconstruction of Bone Tissue Using CAS_Annotate and CAS_Navigate. Journal of Biomedical Engineering and Medical Imaging, Volume 4, No 5, Oct (2017), pp 19-26

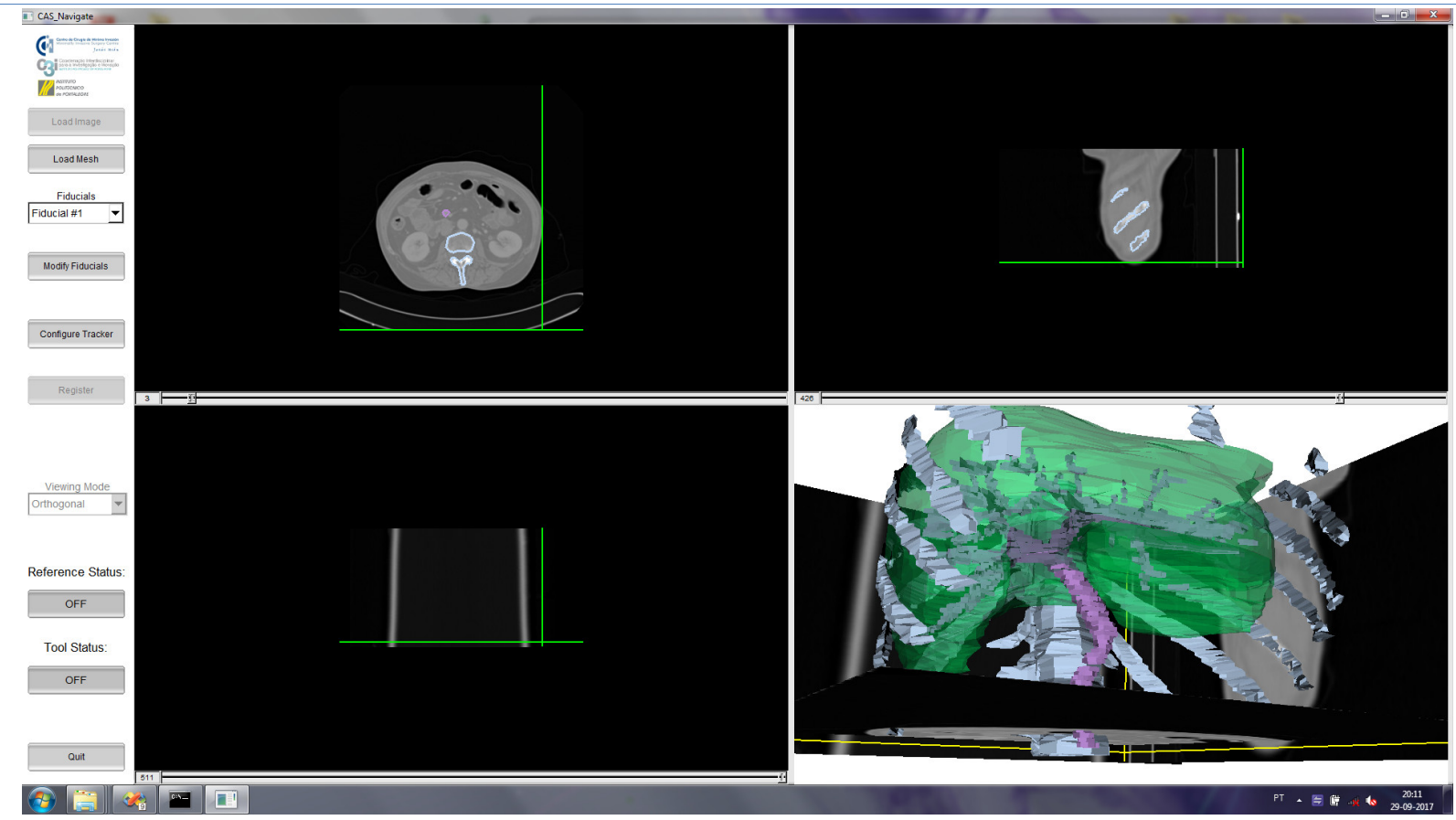

Figure 7 - Volume-based reconstructed model (veins) and surface based reconstructed models (liver+bones) visualization in CAS_Navigate

Table 2 - Model complexity

\begin{tabular}{|c|l|c|c|c|c|}
\hline$\#$ & \multicolumn{1}{|c|}{ Model } & $\begin{array}{c}\text { Type of } \\
\text { Reconstruction }\end{array}$ & Resolution & \#Triangles & \#Vertices \\
\cline { 1 - 4 } 1 & Liver2 (composite surface) & surface & low & 3248 & 1626 \\
\hline 2 & Liver2b (composite surface) & surface & low & 550 & 277 \\
\hline 3 & Vascular network & volume & low & 7572 & 4388 \\
\hline 4 & Rib cage (low_res_surface_model) & surface & low & 14090 & 7083 \\
\hline 5 & Rib cage (high_res_block_model) & volume & high & 498116 & 250488 \\
\hline & Total A (1+2+3+4) & - & - & 25460 & 13374 \\
\hline & Total B (1+2+3+5) & - & - & 509486 & 256779 \\
\hline
\end{tabular}

\section{Conclusions}

The volume-based reconstruction by definition limits the maximum horizontal and vertical geometric error of the model to be the space between slices. This is particularly useful, as drastic polygon shape changes in adjacent slices might generate some rendering artifacts in a surface-based reconstruction. The surface reconstruction required 50 more minutes than the volume-based reconstruction for the user to manually define where the 19 objects started and ended. Further defining where each vertebra starts and ends could potentially solve the rendering artifacts in the surface approach, but requires more user time, and it could be potentially difficult to establish as vertebrae are not scan aligned.

Finally, for the purposes of navigation, inspection of vascular structures of a liver, the artifacts did not pose a problem as they are small and localized. Either volume-based or surface-based reconstruction gave the geometric spatial context desired to inspect the liver and vascular networks. 


\section{ACKNOWLEDGMENTS}

This work was partially funded by Programa de Cooperación Transfronteriza España-Portugal (POCTEP), Fondo Europeo de Desarrollo Regional (FEDER) 0401_RITECA_II_4_E.

\section{REFERENCES}

[1] Meyers D, Reconstruction of Surfaces from Planar Contours. PhD Thesis. University of Washington, 1994.

[2] ITK, ITK-Snap, http://www.itksnap.org, 2017.

[3] Oliveira, J. F. , J. L. Moyano-Cuevas, J. Blas, H. Capote, and F. M. S. Margallo, Preoperative and Intraoperative Spatial Reasoning Support with 3D Organ and Vascular Models: Derived from CT Data using VTK and IGSTK, International Journal of Creative Interfaces and Computer Graphics, vol. 6(2), pp. 56-82, July-December, 2015.

[4] Sliver, http://www.sliver07.org, 2017.

[5] The Visualization Toolkit, http://www.vtk.org, 2017.

[6] Pianykh, O. S., Digital Imaging and Communications in Medicine (DICOM) A Practical Introduction and Survival Guide, $2^{\text {nd }}$ Edition, Springer, ISBN 978-3-642-10849-5, 2012.

[7] The Fast Light Toolkit (FLTK), www.fltk.org, 2017.

[8] Eberly D, Triangulation by Ear Clipping, http://www.geometrictools.com, 2008.

[9] Christiansen HN, Sederberg TW, Conversion of complex contour line definitions into polygonal element mosaics. Computer Graphics XIII. 2(August, 1978):187-192, 1978.

[10] Oliveira, J. F., Advantages and Disadvantages of using Third-party software in the development of the CAS_Annotate and CAS_Navigate Medical Applications, Journal of Biomedical Engineering and Medical Imaging, 3(6), ISSN: 2055-1266, 2016.

[11] The Stanford 3D Scanning Repository, Stanford University, http://graphics.stanford.edu/data/3Dscanrep/, 2017.

[12] The Image-Guided Surgery Toolkit, http://www.igstk.org, 2017.

[13] Insight Segmentation and Registration Toolkit (ITK), https://itk.org, 2017. 